\title{
Caracterización de la fatiga muscular en músculos con diferente metabolismo fibrilar. Un estudio experimental in vitro
}

\author{
Marta Sierra $^{1}, \mathrm{M}^{\mathrm{a}}$ Jesús Muñoz ${ }^{2}$, Jorge Grasa ${ }^{1}$ \\ ${ }^{1}$ Applied Mechanics and Bioingeneering (AMB) \\ ${ }^{2}$ Laboratorio de Genética Bioquímica de la Universidad de Zaragoza (LAGENBIO) \\ Instituto de Investigación en Ingeniería de Aragón (I3A). \\ Universidad de Zaragoza, Mariano Esquillor s/n, 50018, Zaragoza, Spain. \\ Tel. +34-976762707, Fax +34-976762043, e-mail: msierra@unizar.es
}

\begin{abstract}
Los objetivos de este trabajo son: la caracterización de las propiedades contráctiles de los músculos EDL y Sóleo de conejo y el estudio de la fatigabilidad del tejido. El estudio permitió obtener las propiedades contráctiles del tejido, la evolución de la fuerza y concentración de glucógeno durante el protocolo de desarrollado.
\end{abstract}

\section{Cuerpo}

El tejido muscular está compuesto por células o fibras musculares que presentan características diferentes según la función del músculo en el que se encuentran. Así, las fibras tipo II son fibras rápidas, que generan una gran fuerza en corto espacio de tiempo. En el caso de las fibras tipo I o fibras lentas, la respuesta está ralentizada y el nivel de fuerza es menor pero son capaces de mantener este nivel más tiempo, de forma que la aparición de la fatiga se ralentiza, al contrario que con las fibras tipo II.

La fatiga muscular se describe como el estado en el que el músculo no puede mantener su capacidad de respuesta a un estímulo. Hoy en día, la aparición de la fatiga se considera un fenómeno multifactorial en el que actúan: la falta de oxígeno, la acumulación de iones o el agotamiento de la reserva de energía (glucógeno), entre otros.

Con el objetivo de caracterizar los componentes mecánicos y bioquímicos de la fatiga en dos músculos con composición de fibras diferente, se escogieron el músculo Extensor Digitorum Longus (EDL), con un alto porcentaje de fibras rápidas y el músculo Sóleo, predominantemente lento, ambos de la extremidad inferior del conejo.

\section{Material y Métodos}

Este estudio fue realizado en 10 conejos White New Zealand machos y peso $2 \pm 0,2 \mathrm{~kg}$. Los animales se mantuvieron en una sala con temperatura controlada $\left(22^{\circ}\right)$, con ciclos luz-oscuridad de doce horas y libre acceso a agua y comida. Todos los experimentos fueron aprobados por el Comité Ético para el Uso de Animales de Experimentación de la Universidad de Zaragoza.

Los animales se dividieron en dos grupos $(n=5)$ para estudiar la fatiga del EDL y del Sóleo y en todos ellos se ensayó la extremidad inferior derecha.. Los animales se anestesiaron con Medetomidina $(0,5 \mathrm{mg} / \mathrm{kg})$, Butorfanol $(0,4 \mathrm{mg} / \mathrm{kg})$ y Ketamina $(25 \mathrm{mg} / \mathrm{kg})$. Una vez que el EDL y el Sóleo de la extremidad posterior derecha fueron extraídos, los animales se eutanasiaron con una sobredosis de pentobarbital sódico.

El baño de órganos fue diseñado por nuestro equipo con las características necesarias para llevar a cabo este estudio. Consta de una cubeta de metacrilato de $20 \times 20 \times 20 \mathrm{~cm}$ con un sistema de anclaje en la base inferior que permite fijar el tendón distal del músculo. Así mismo, dispone de una estructura superior, también de metacrilato, en la que se insertan dos electrodos de platino que pueden ser colocados a la distancia óptima para conseguir el correcto estímulo de la muestra. Durante la realización de las pruebas experimentales, se colocó en el baño de órganos una solución glucosada que aportó los nutrientes necesarios a la muestra durante todo el ensayo y que se mantuvo a la temperatura óptima $\left(25^{\circ}\right)$ gracias al acoplamiento de una bomba con calentador. La cubeta se dispuso en una máquina universal de ensayos (Instron®), de manera que el tendón proximal del músculo se fijó a la máquina y el músculo quedó en disposición vertical, alineado con el eje de acción de la célula de carga.

Previamente, se realizaron barridos para obtener los parámetros óptimos de voltaje, frecuencia y tiempo de pulso con los que se obtiene la máxima fuerza para cada tipo de músculo. En el caso del EDL, estos parámetros fueron: $100 \mathrm{~V}, 100 \mathrm{~Hz}$ y $200 \mathrm{~ms}$, con un intervalo de 10,2 segundos entre pulso y pulso. En el caso del Sóleo: $100 \mathrm{~V}, 100 \mathrm{~Hz}$ y 1,5 segundos con un intervalo de 11,5 segundos. Además, en cada músculo ensayado se relizó previamente un barrido 
de longitud para determinar la longitud óptima a la que el músculo ejerce la máxima fuerza. A continuación, se procedió a la estimulación, el primer grupo, tanto de EDL como de Sóleo $(n=5)$ durante 60 minutos, para alcanzar la fatiga total, y el segundo $(n=5)$ durante 25 minutos, con el objetivo de determinar la cantidad de glucógeno del músculo a ese tiempo. Al finalizar cada experimento en ambos grupos, los músculos junto con sus contralaterales fueron congelados en nitrógeno líquido para determinar mediante la técnica de la Antrona[1] la concentración de glucógeno.

\section{Resultados}

En el caso del EDL $(2,22 \pm 0,12$ g) $(\mu \pm \varphi)$, la fuerza media observada fue de 16,85 $\pm 3,06 \mathrm{~N}$ Se observa una caída significativa de la fuerza al final de la estimulación (Fig.1), con un nivel de 0,803 \pm 0,29N (4,76\%). Esta caída no es uniforme, a los 25 minutos de estimulación, la fuerza cae hasta un nivel de 2,705 $\pm 1,28 \mathrm{~N}$ (16\%). Entre los valores de fuerza a los 25 y a los 60 minutos no se han observado diferencias significativas. En el caso del glucógeno, la cantidad media en los musculos control fue de 132,77 $\pm 37,13 \mathrm{mg} / \mathrm{g}$ de músculo. Esta cantidad cayó hasta el 49,12 \pm 18,92 mg/g de músculo (36,99\%) a los 25 minuto de estimulación y hasta 26,12 $\pm 12,55 \mathrm{mg} / \mathrm{g}$ de músculo (19,67\%) a los 60 minutos de ensayo. Al igual que en la fuerza, no se observa diferencias significativas entre las cantidades a 25 y a 60 minutos. Se realizó un test de regresión entre los niveles de fuerza y la cantidad de glucógeno a lo largo del tiempo y se obtuvo una correlación del 84\%.

En el caso del Sóleo $(1,79 \pm 0,18$ g), (Fig. 2), la fuerza máxima fue de $7,8 \pm 2,07 \mathrm{~N}$. Al final de los 60 minutos de estimulación, se observa una caída significativa de la fuerza, con un nivel de 1,28 \pm 0,33N (29,74\%). Esta caída, como en el EDL, no es uniforme, a los 25 minutos la fuerza cae hasta 2,32 $\pm 0,80 \mathrm{~N}(16,41 \%)$. Entre los valores de fuerza a los

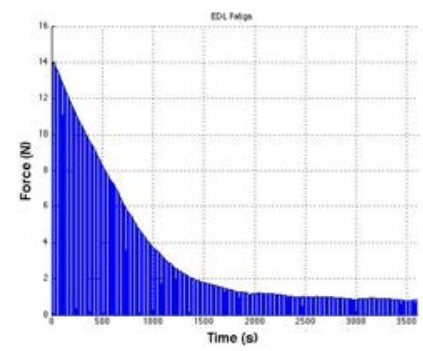

Fig. 1. Evolución de la fuerza ejercida por el músculo EDL.
25 y los 60 minutos no se han observado diferencias significativas. En el caso del glucógeno, la cantidad media en los musculos Sóleos control fue de 45,73 $\pm 4,85 \mathrm{mg} / \mathrm{g}$ de músculo. Esta cantidad cayó hasta el 21,25 \pm 3,25 mg/g de músculo (47,22\%) a los 25 minuto de estimulación y hasta 21,96 $\pm 3,91 \mathrm{mg} / \mathrm{g}$ de músculo $(48,02 \%)$ a los 60 minutos. No se observan diferencias significativas entre las cantidades a 25 y a 60 minutos. Se realizó un test de regresión entre los niveles de fuerza y la cantidad de glucógeno a lo largo del tiempo y se obtuvo una correlación del 86,6\%.

\section{Conclusiones}

Los resultados experimentales obtenidos, evidencian la diferencia de comportamiento de los músculos con diferente composición de fibras. En el caso del EDL (fibras rápidas) el nivel de fuerza es mayor pero también la aparición de la fatiga se produce antes, mientras que en el Sóleo, el nivel de fuerza es más bajo pero es capaz de mantenerla durante más tiempo. Esta diferencia también queda reflejada en la caída del glucógeno, mucho más pronunciada en el EDL que en el Sóleo.

Los resultados obtenidos permitirán el desarrollo y validación de un modelo computacional que simule el comportamiento de fatiga muscular en músculos con predominancia de fibras lentas y en aquellos con mayor porcentaje de fibras rápidas. De la misma forma, el dispositivo experimental desarrollado permitirá el estudio en profundidad de enfermedades neurodegenerativas que afectan al músculo, así como los posibles tratamientos desarrollados de manera experimental.

\section{REFERENCIAS}

[1]. CARROLL N.V., LONGLEY R.W., and ROE J.H. The determination of glycogen in liver and muscle by use of anthrone reagent. The journal of biological chemistry. 1956, 220(2), 583-593.

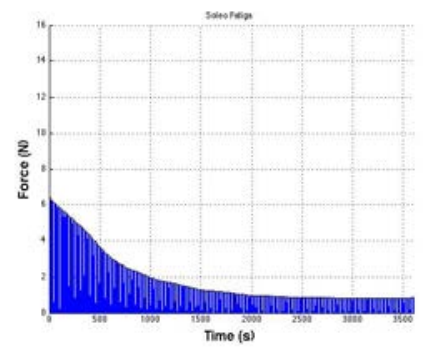

Fig. 2. Evolución de la fuerza ejercida por el músculo Sóleo. 